\title{
High-Frequency Signature-Based Fault Detection for Future MV Distribution Grids
}

Yaswanth Nag Velaga ${ }^{1}$, Kumaraguru Prabakar ${ }^{1}$, Akanksha Singh ${ }^{1}$, and Pankaj K. Sen ${ }^{2}$

${ }^{1}$ National Renewable Energy Laboratory (NREL), Golden, Colorado

${ }^{2}$ Colorado School of Mines, Golden, Colorado

2020 Industrial and Commercial Power Systems Technical Conference

June 29 - July 28,2020 


\section{DER Impact on Protection and Challenges}

\section{US Generation Sources}

\begin{tabular}{|ll|}
\hline wind & $7.3 \%$ \\
hydro & $6.6 \%$ \\
solar & $1.8 \%$ \\
biomass & $1.4 \%$ \\
geothermal & $0.4 \%$ \\
\hline
\end{tabular}
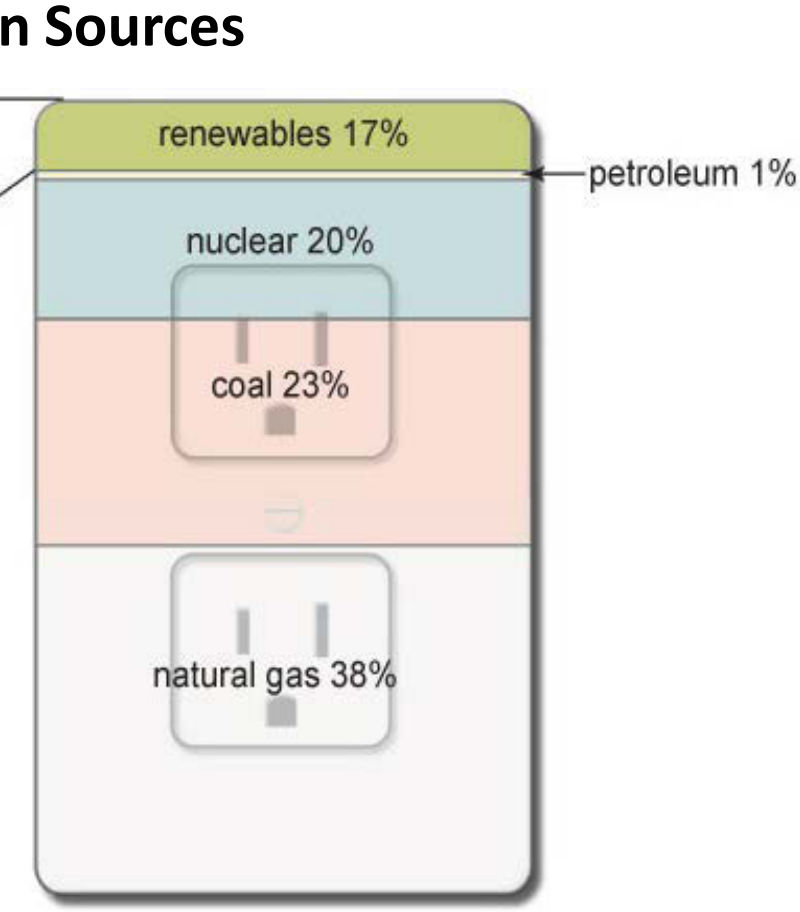

High Pen PV/DER

- Low fault current

- Reverse power flow

- Bottleneck for high penetration

- Intermitten DER resourc

Phasor based

- One full cycr. observation window (slow)

- May need adaptive or multiple settings
Resiliency

- High-impact, lowfrequency events

- Damage prevention Traveling-wave based covery protection is applied to the future distribution grid

- Future distribution network needs high speed fault detection and isolation 


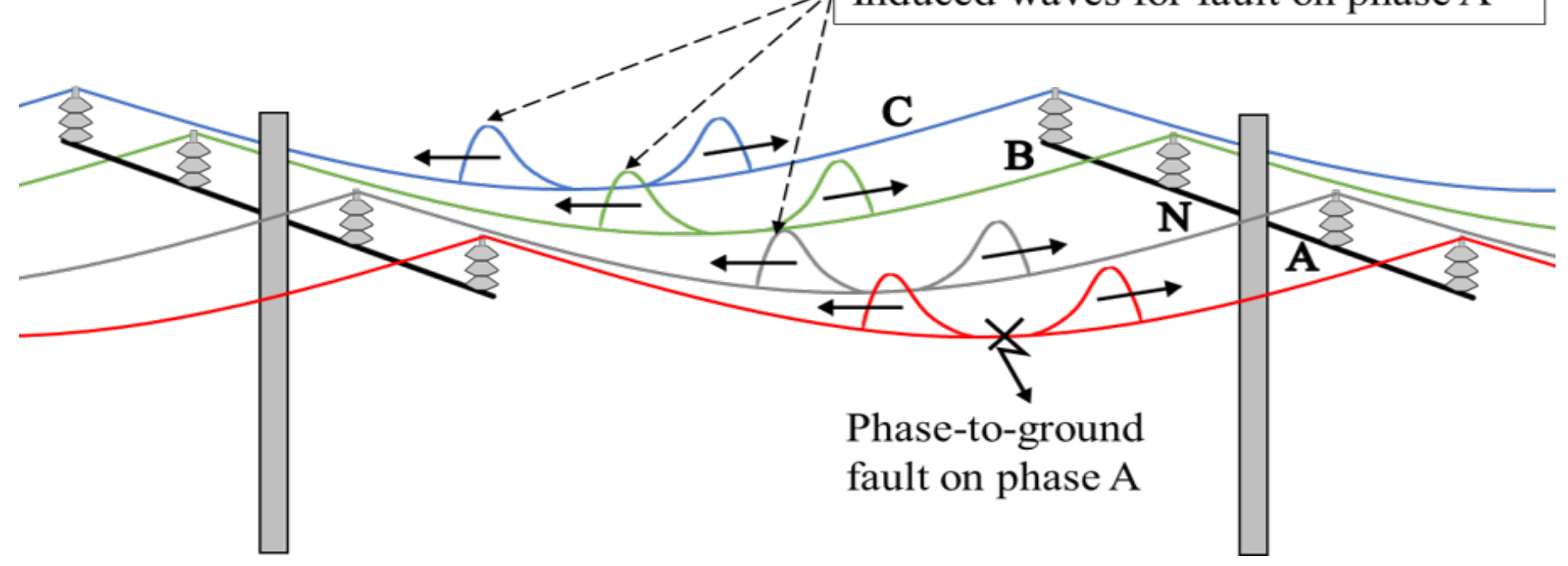

$>$ Any disturbances in the circuit caused by fault, switching, lightning creates a traveling-wave (TW) transient.

$>$ Waves travel at close to the speed of light $(186,282 \mathrm{mi} / \mathrm{s})$.

$>$ Used in insulation failure and surge protection design. 


\section{Advantages}

$>$ Independent of fault currents

$>$ Not affected by CT saturation, power swings, line compensation

$>$ Application to single and two phases

$>$ Faster fault detection

\section{Challenges}

$>$ Frequent taps

$>$ High attenuation

$>$ Presence of transformers,

capacitors

$>$ Requirements for CT and PT are high

High-frequency signatures generated as a result of TW are used to detect and locate a fault 


\section{Line Models for EMTP}

Frequency-dependent (FD) model is the most accurate line model to study the high frequency transients.

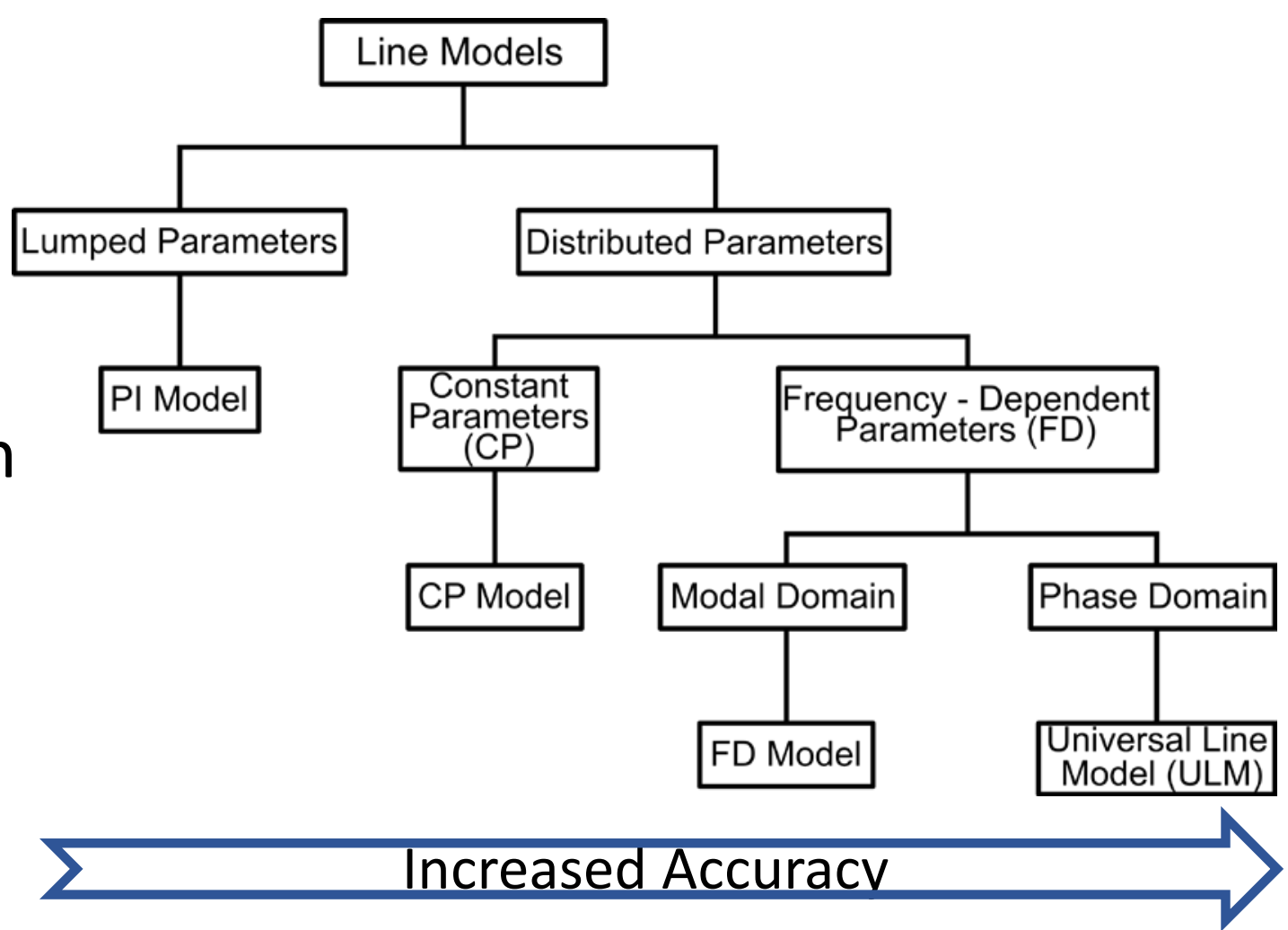




\section{Line Models Frequency Response}
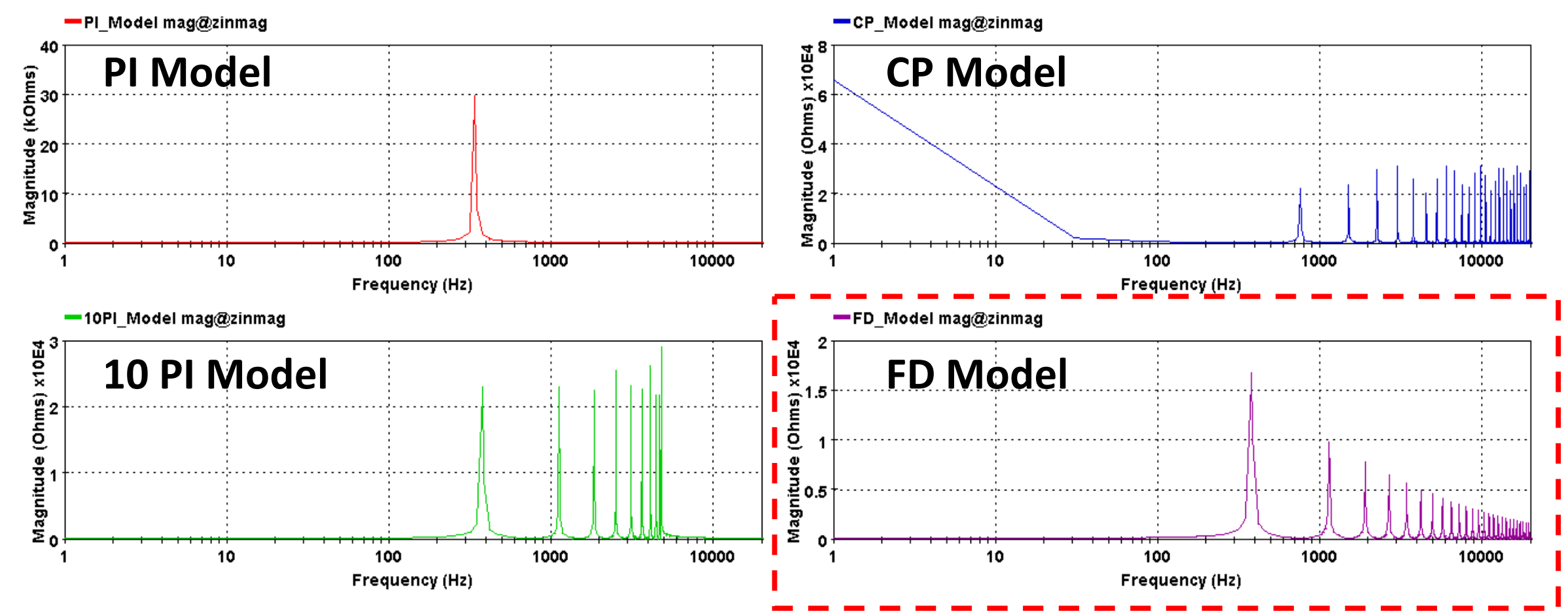


\section{Traveling-Waves Simulation}

$>$ Modified IEEE 13-bus test system is developed as $13.8 \mathrm{kV}$. medium voltage (MV) distribution network in EMTP-RV

$>$ Understand the signature of the transients in the complex network.

$>$ Ideal CT and PT characteristics are assumed.

$>$ Frequency dependent (FD) line model is crucial for EMTP simulations.

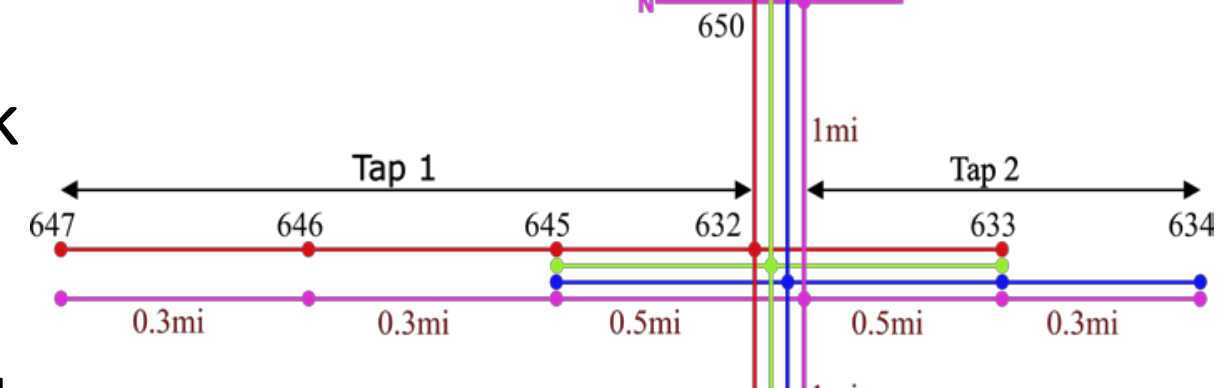




\section{Distribution Test Case}

Modified IEEE test system at $13.8 \mathrm{kV}$. Two overhead lines are replaced by underground cables between 645 \& 632, 671B \& 675.

$>$ Simulation timestep off $100 \mathrm{~ns}$ is used to study the different cases in test system.

Voltage and current probes at every bus are sampled at $10 \mathrm{MHZ}$ to record high frequency waves.

\section{7}

$0.3 \mathrm{mi}$
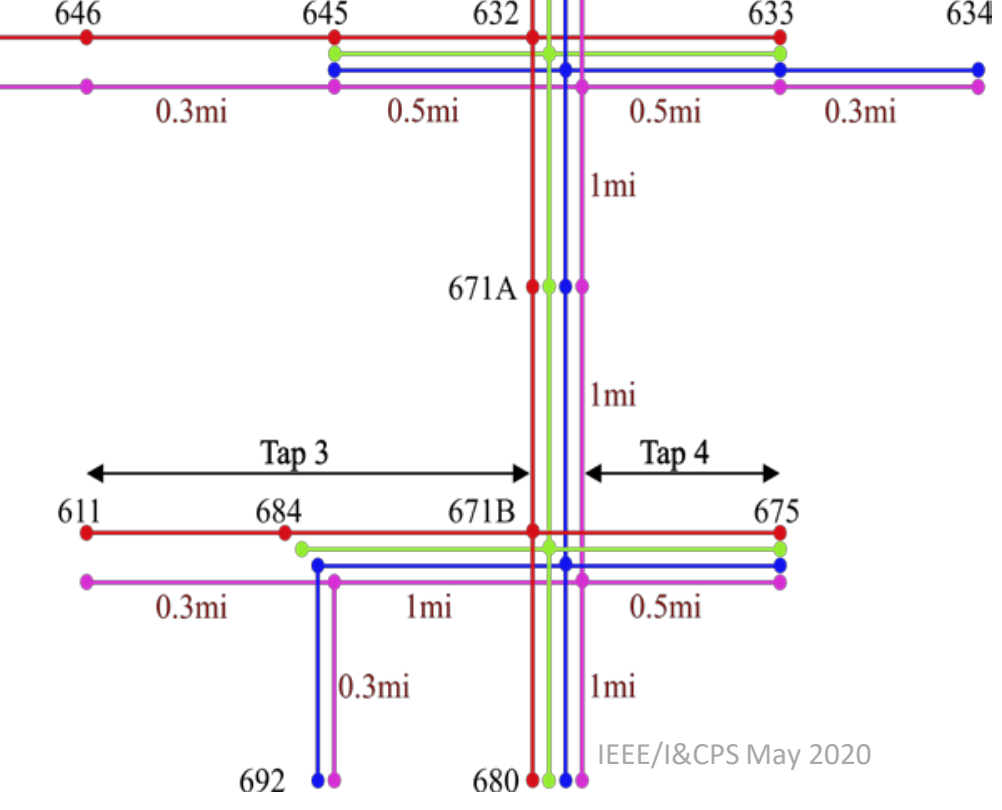


\section{Traveling-Wave Timing \\ Diagram}

$\mathrm{OH}$ - Overhead Line

UG - Underground Cable

$>$ Indicated times are the wave propagation time from one end to other end of line.

To estimate arrival wave times at buses, add the times in the path between fault and selected bus.

Alpha mode velocities are used to calculate the propagation time.

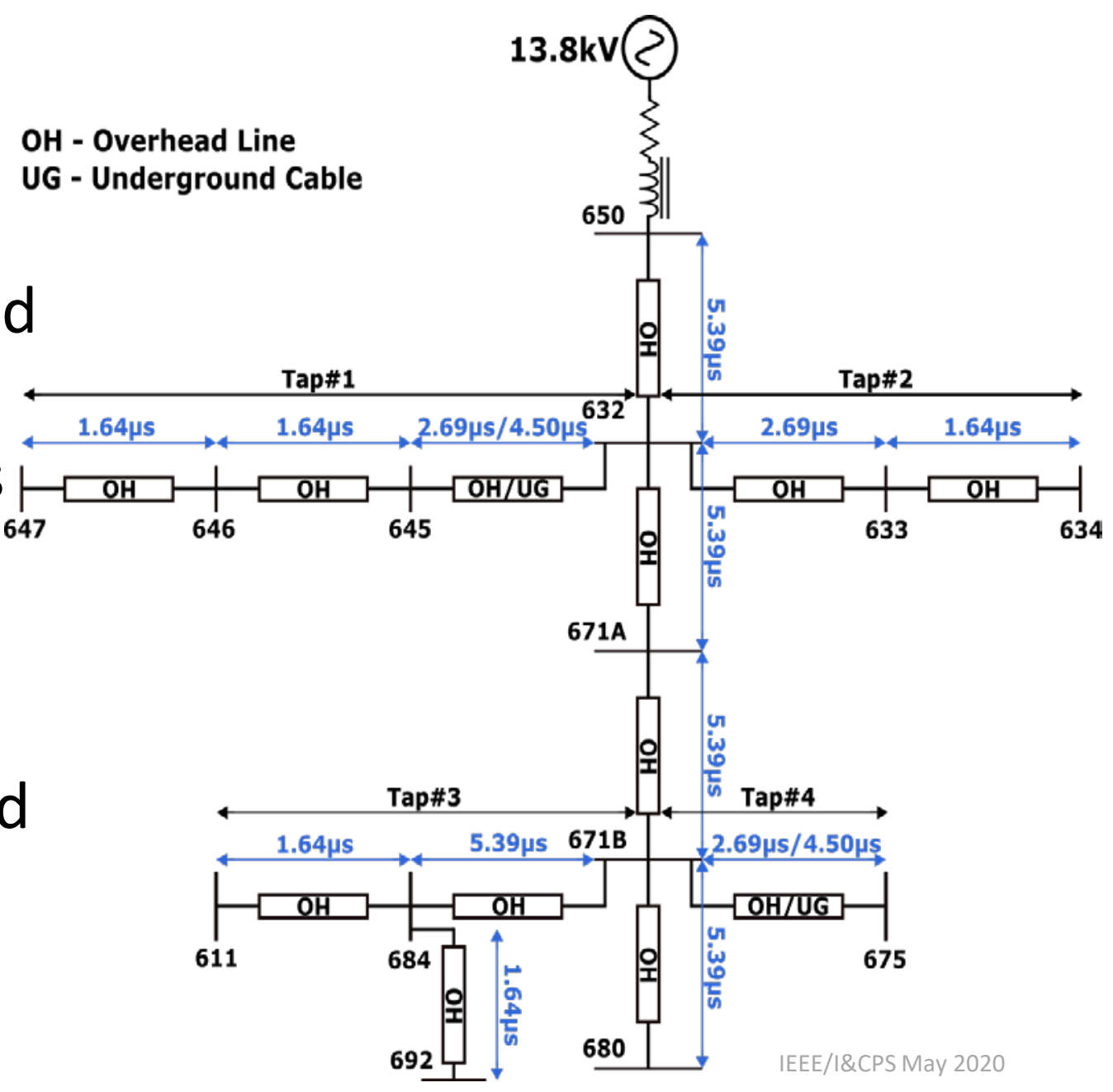


Overhead Lines with SLG Fault on Bus 680

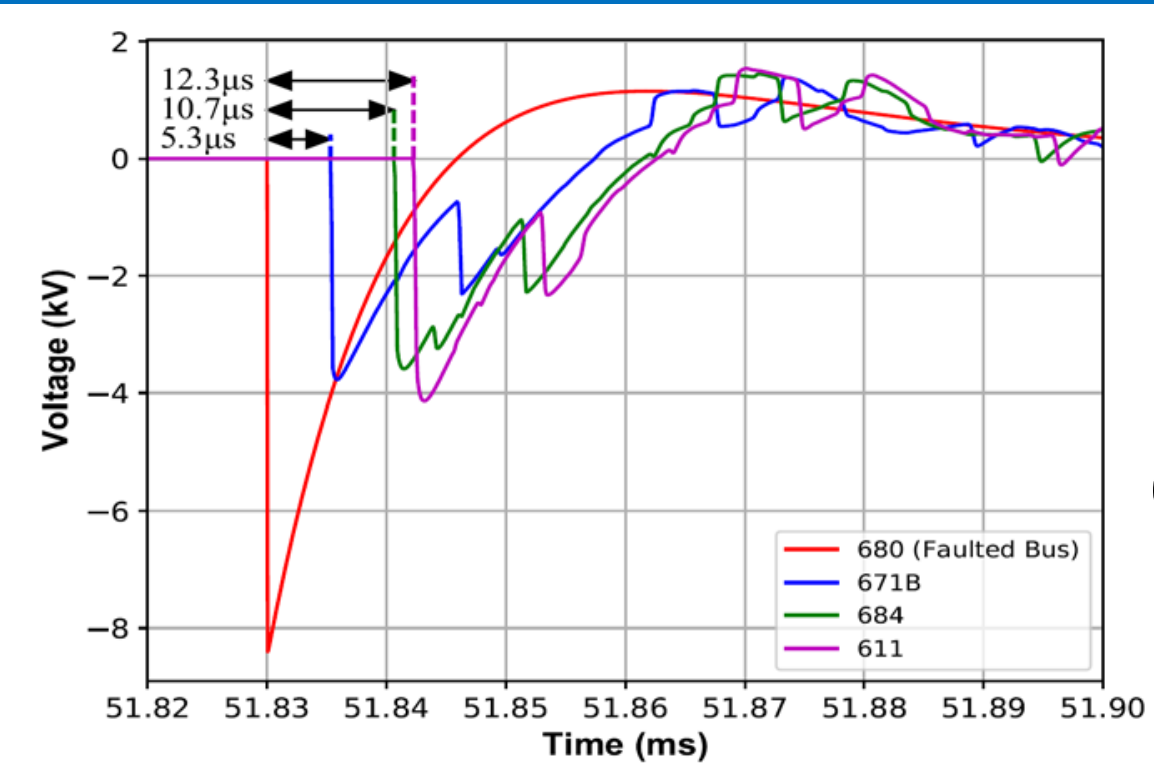

OH - Overhead Line

UG - Underground Cable

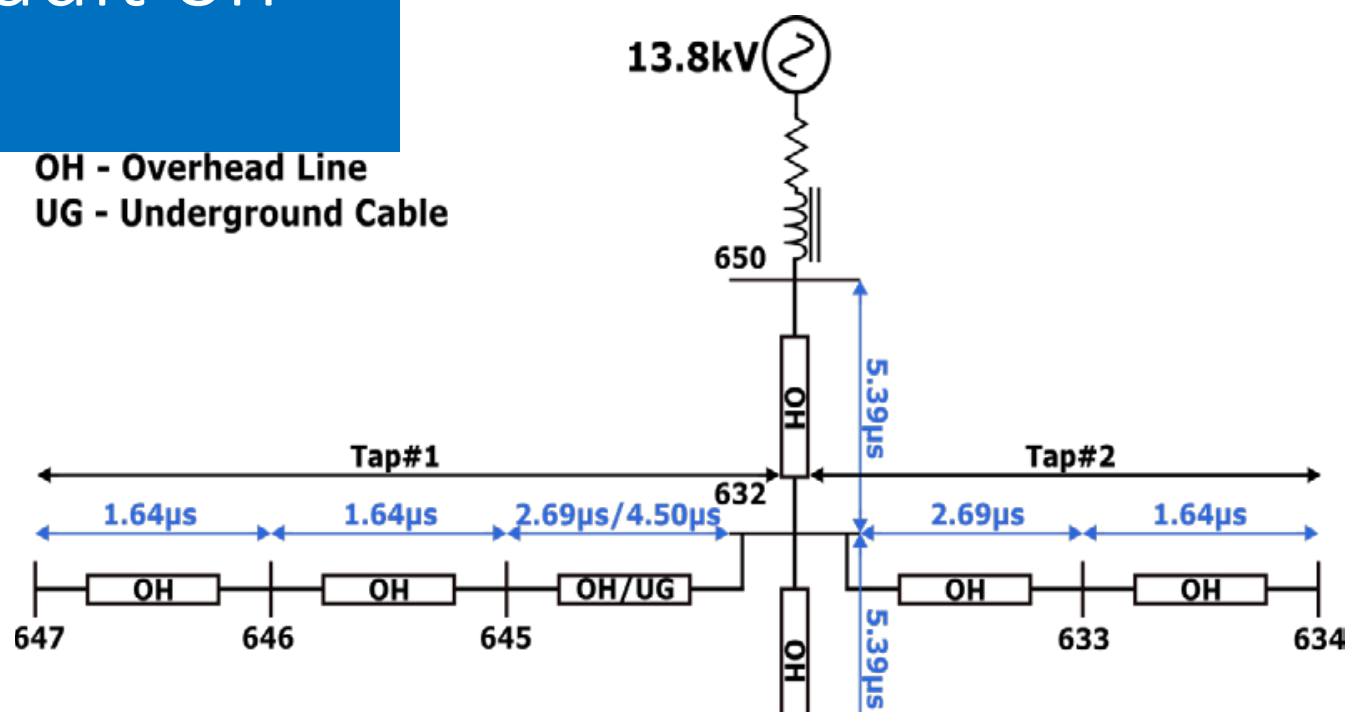

$>$ Estimated wave arrival times match the simulated times in tap 3 $>$ Multiple reflections due to taps

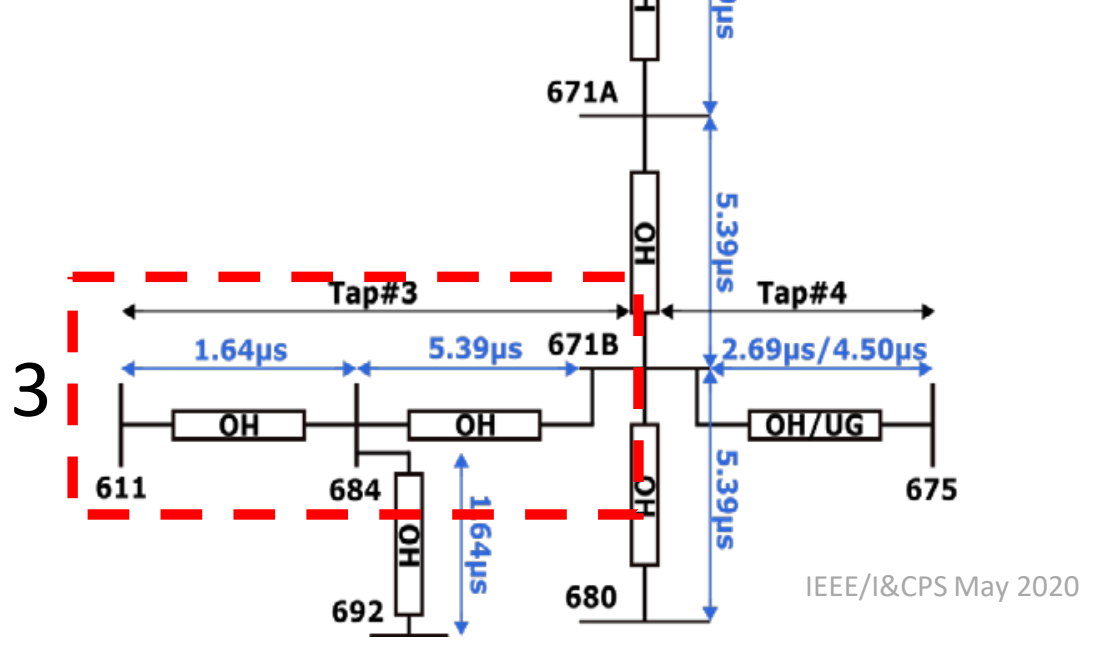


OH Lines with Line-to-Line Fault

\section{on Bus 680}

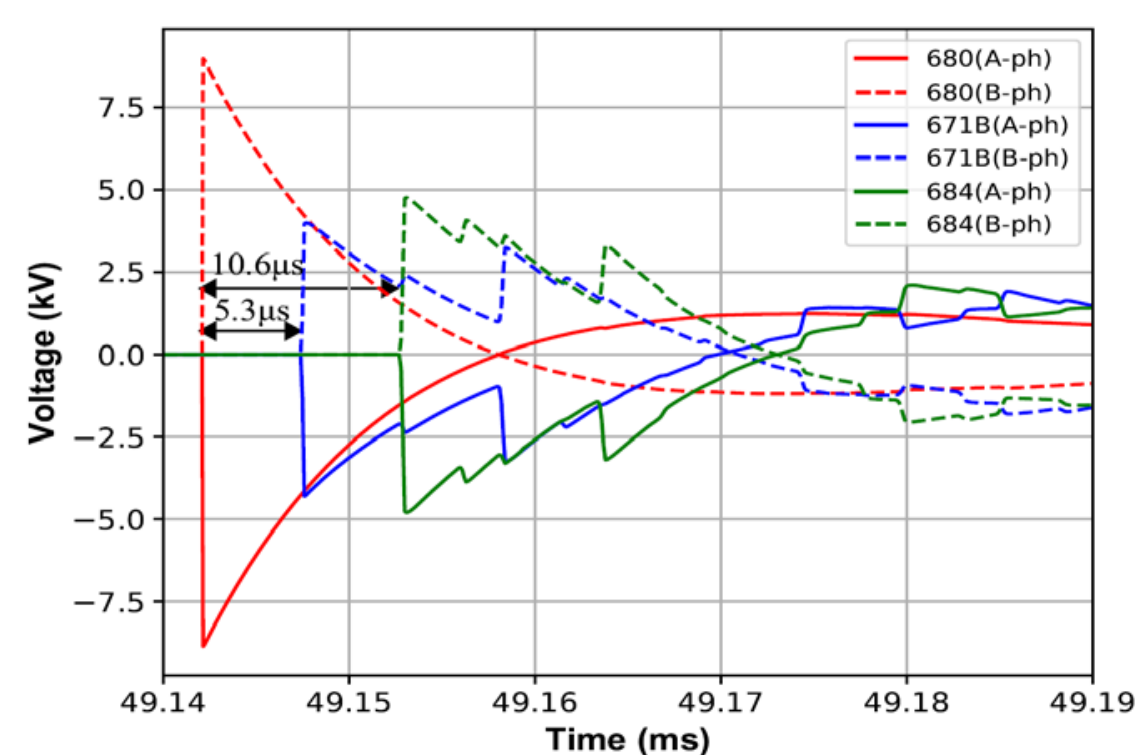

Waves launched at the fault depends on voltage difference

Similar characteristic to LL fault currents
$\mathrm{OH}$ - Overhead Line

UG - Underground Cable
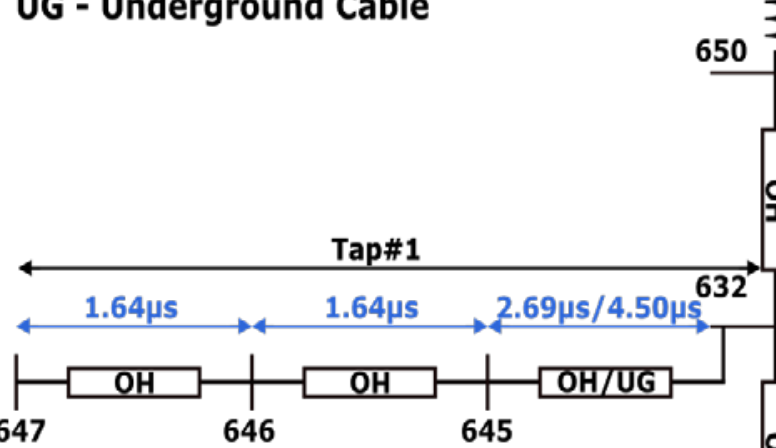

647

645

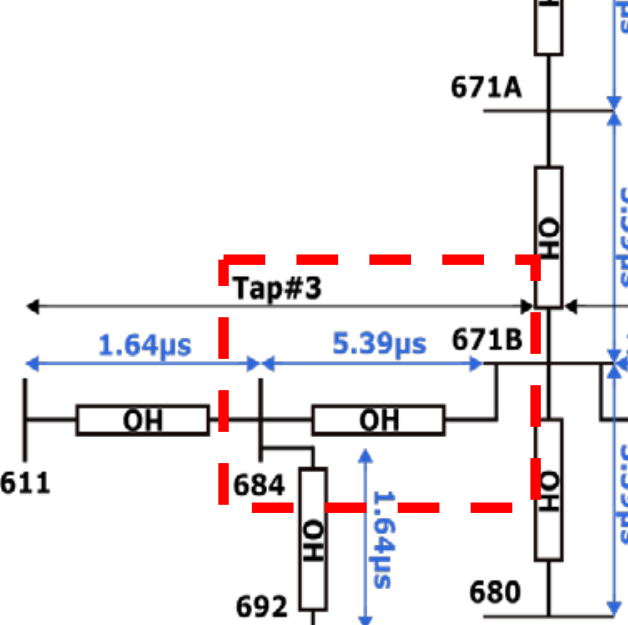




\section{Future Work}

$>$ Different fault types such as high impedance, arcing fault will be studied using the test system.

$>$ Frequency signatures will be developed using advanced signal processing techniques.

$>$ High frequency models of transformer and DER will be developed and validated through testing.

$>$ Results will be validated through field data. 


\title{
Thank you Email: yaswanthnag.velaga@nrel.gov
}

\author{
NREL/PR-5D00-77133
}

\begin{abstract}
This work was authored in part by the National Renewable Energy Laboratory, operated by Alliance for Sustainable Energy, LLC, for the U.S. Department of Energy (DOE) under Contract No. DE-AC36-08GO28308. Funding provided by U.S. Department of Energy Office of Energy Efficiency and Renewable Energy (EERE) under Solar Energy Technologies Office (SETO) Agreement Number 34237. The views expressed in the article do not necessarily represent the views of the DOE or the U.S. Government. The U.S. Government retains and the publisher, by accepting the article for publication, acknowledges that the U.S. Government retains a nonexclusive, paid-up, irrevocable, worldwide license to publish or reproduce the published form of this work, or allow others to do so, for U.S. Government purposes.
\end{abstract}

